\title{
Induction of cytotoxic $T$ lymphocytes against human cancer cell lines using dendritic cell-tumor cell hybrids generated by a newly developed electrofusion technique
}

\author{
KENICHIRO IMURA $^{1 *}$, YUJI UEDA ${ }^{1 *}$, TAKASHI HAYASHI ${ }^{2 *}$, TSUYOSHI ITOH ${ }^{1}$, KEIJI SHIMIZU $^{1}$, \\ HIDEMASA TAMAI $^{1}$, YUTARO YANO ${ }^{1}$, KEI NAITO ${ }^{1}$, JUNJI KOHARA ${ }^{1}$, KAZUKI NAKANE $^{3}$, \\ YUKO MATSUURA $^{3}$, ATSUKO TAKEDA ${ }^{3}$, TAKAHISA TAKEDA ${ }^{3}$, \\ KEIICHI KAWAI ${ }^{3}$ and HISAKAZU YAMAGISHI ${ }^{1}$ \\ ${ }^{1}$ Department of Surgery, Division of Digestive Surgery, Kyoto Prefectural University of Medicine, 465 Kajii-cho, \\ Kawaramachi-Hirokoji, Kamigyo-ku, Kyoto 602-8566; ${ }^{2}$ Department of Surgery, Takeda Hospital, \\ Shiokoji-cho 841-5, Shimogyo-ku, Kyoto 600-8558; ${ }^{3}$ Bio-health Center, Takeda Hospital, \\ Keiaikosan-Kyoto Bldg., Kankoboko-cho 87, Shimogyo-ku, Kyoto 600-8009, Japan
}

Received March 8, 2006; Accepted May 2, 2006

\begin{abstract}
Recently, dendritic cells (DCs) and DC-tumor cell hybrids (DC-tumor hybrids) have been used for cancer vaccine therapy in a clinical trial. DC-tumor hybrids combine the potent antigen-presenting capacity of DCs with the ability to present all tumor antigens expressed on tumor cells to T cells. We used DC-tumor hybrids as stimulator cells to induce tumor-specific cytotoxic T lymphocytes (CTLs) in vitro. DC-tumor hybrids were generated from human monocytederived DCs and human cancer-cell lines (GT3TKB, lung cancer; GCIY, gastric cancer) by our newly developed electrofusion technique, established and refined with the use of mouse cells. To evaluate the capacity of DC-tumor hybrids generated by our method to induce tumor antigen-specific CTLs, we performed a cytotoxic assay and an interferon- $\gamma$ release assay using CD8-dominant effector lymphocytes induced by them. DC-tumor hybrids more effectively induced tumor-specific primary $\mathrm{T}$-cell response than did stimulation with DCs co-cultured with irradiated tumor cells overnight, irradiated tumor cells alone, or a mixture of DCs and irradiated tumor cells. DC-tumor hybrids were generated at a high fusion rate by our electrofusion technique. When
\end{abstract}

Correspondence to: Dr Yuji Ueda, Department of Surgery, Division of Digestive Surgery, Kyoto Prefectural University of Medicine, 465 Kajii-cho, Kawaramachi-Hirokoji, Kamigyo-ku, Kyoto 602-8566, Japan

E-mail: yueda@koto.kpu-m.ac.jp

${ }^{*}$ Contributed equally

Key words: dendritic cell, cytotoxic $\mathrm{T}$ lymphocyte, human cancer cell line, cancer immunotherapy, electrofusion, DC-tumor hybrid
CTLs were induced by DC-tumor hybrids in vitro, the high fusion rate did not contribute to the induction of CTLs with increased tumor-specific cytotoxicity. The addition of interleukin-12 to the culture medium did not augment the cytotoxicity of CTLs. Overall, our results suggest that DCtumor hybrids effectively induce human tumor-specific CTLs and may thus be applicable for clinical trials of adoptive immunotherapy.

\section{Introduction}

The rationale for cancer vaccine therapy is the induction of tumor-specific cytotoxic T lymphocytes (CTLs) by vaccine in vivo. Recently, many clinical trials have evaluated vaccine therapy with the use of dendritic cells (DCs) because of their very high antigen-presenting capacity. DCs are the only antigen-presenting cells (APCs) that can stimulate native $\mathrm{T}$ cells, making them useful tools for the induction of CTLs (1-3).

In early attempts to use DCs for cancer vaccine therapy, peptides and proteins derived from known tumor-associated antigens (TAAs) were pulsed to DCs (4-7), or virus vectors integrating specific TAAs were transduced to DCs (8-10). Theoretically, these approaches made sense, but were of limited value clinically. It appeared that immunotherapy targeting a single TAA was not adequately effective clinically against tumors expressing many known and unknown TAAs. These disappointing results led to several attempts to develop treatment techniques using all TAAs expressed on tumor cells. Tumor lysates or apoptotic bodies of tumor cells were pulsed to DCs (11-14), or DCs were loaded with RNA extracted from tumor cells (15). Another related technique involved the use of fusion cells (FCs) generated from DCs and tumor cells (DC-tumor hybrids) as cancer vaccine. With this technique, untreated tumor cells are fused with DCs. Consequently, all TAAs expressed on tumor cells can be naturally and efficiently presented by DCs. In this study, we generated FCs from peripheral blood monocyte-derived DCs 
and human cancer cell lines and evaluated the tumor-specific CTL induction capacity of these FCs in vitro. Many methods for cell fusion have been reported. In 1979, B-cell lymphoma cells and myeloma cells fused with the use of polyethylene glycol (PEG) were reported to retain the characteristics of each cell type (16). Owing to the toxicity of PEG and the low fusion rate, we used electrofusion in the present study.

We have previously studied the cytotoxic activity of FC-based cancer vaccine therapy in a mouse model. Subcutaneous tumor and lung metastases were found to regress after a single nodal injection of FCs with interleukin (IL)-12 as an adjuvant (17-21). However, clinical studies of hybrid cell vaccinations (FCs) showed no evidence of the dramatic response seen in mouse models of cancer; response was negligible or minor, even after several inoculations of vaccine $(22,23)$. Poor clinical response was attributed to the lack of concurrent treatment with adjuvant agents such as IL-12, which was required for an antitumor response in mouse models. Another probable reason for the poor response was that the patients had far-advanced disease, associated with increased production of various immunosuppressive cytokines, such as transforming growth factor (TGF)- $\beta$. Increased levels of immunosuppressive cytokines may have inhibited the antigenpresenting capacity of FCs (24). Progressive disease with elevated levels of immunosuppressive cytokines may thus considerably reduce the efficacy of active immunotherapy using FCs as effectors.

In adoptive immunotherapy, CTLs induced by using FCs as stimulators in vitro are used as effector cells, thereby avoiding the problems associated with active immunotherapy in patients with cancer. We induced CTLs against human cancer cell lines by using FCs as stimulator cells in vitro to examine whether FCs could be used for adoptive immunotherapy. We compared several types of APCs to determine which was best suited for the induction of CTLs. Since FCs were generated at high fusion rates by our modified electrofusion method, we examined whether the activity of induced CTLs was affected by the fusion rate. In addition, we studied whether IL-12, an adjuvant required for in vivo antitumor activity in mouse models, promoted the induction of human CTLs in vitro.

\section{Materials and methods}

Tumor cell lines. Human cancer cell lines, GT3TKB (lung squamous cell carcinoma) and GCIY (gastric adenocarcinoma), were obtained from RIKEN Bio Source Center (Tsukuba, Ibaragi, Japan). Each cell line was maintained in vitro at $37^{\circ} \mathrm{C}$ in RPMI-1640 (Nikken Biomedical Center, Kyoto, Japan), supplemented with $10 \%$ heat-inactivated fetal bovine serum (FBS; Thermo Trace Ltd., Melbourne, Australia), 2 mM L-glutamine, and $2 \mu \mathrm{g} / \mathrm{ml}$ gentamycin (Schering-Plough, Osaka, Japan).

DC preparation. DCs were induced from peripheral-blood mononuclear cells (PBMCs). PBMCs were harvested by leukapheresis from a healthy donor (male, aged $\sim 30$ years) who gave written informed consent for the procedure. The cells were incubated in X-VIVO20 medium (Cambrex Bio Science Walkersville, Inc., Walkersville, MD) in $75-\mathrm{cm}^{2}$ flasks (Sumiron, Tokyo, Japan) overnight. Non-adherent peripheral blood lymphocytes (PBLs) were removed and frozen for later use as a cell source for CTL induction. Monocyte-rich adherent cells were cultured in RPMI-1640 supplemented with $2 \%$ heat-inactivated autologous serum containing $40 \mathrm{ng} / \mathrm{ml}$ granulocyte/ macrophage-colony stimulating factor (GM-CSF) (PeproTech House, London, UK) and $20 \mathrm{ng} / \mathrm{mL}$ IL-4 (PeproTech House). After 5 days, 10 $\mathrm{ng} / \mathrm{ml}$ tumor necrotizing factor (TNF)- $\alpha$ (R\&D Systems, Minneapolis, MN) and $1 \mu \mathrm{g} / \mathrm{ml}$ prostaglandin (PG)E 2 (SigmaAldrich, St. Louis, MO) were added for DC maturation (25). On day 7, DCs were harvested and used for electrofusion.

Phenotypic analyses of DCs and effector cells induced by FCs. Flow cytometric analysis (FACS) was performed by FACS Calibur (Becton Dickinson, San Diego, CA), using Cell Quest software (Becton Dickinson). DCs were analyzed for phenotype markers with the following monoclonal antibodies (mAbs): anti-CD14, anti-CD40 (Pharmingen, San Diego, CA) anti-CD80, anti-CD83, anti-CD86, and anti-HLA-DR (class II) (Immunotech, Marseilles, France). Monoclonal Abs for anti-CD4 and anti-CD8 (Pharmingen) were also used for the phenotypic analysis of PBL-derived effector cells induced by FCs.

Electrofusion. Electrofusion of DCs and tumor cells was performed by our newly developed method, as described previously (17). First, DCs and irradiated (30 Gy) tumor cells were mixed at a ratio of $1: 1$ and suspended in fusion medium (4.5\% glucose solution containing $0.1 \mathrm{mM} \mathrm{Ca}\left(\mathrm{CH}_{3} \mathrm{COO}\right)_{2}$, $0.5 \mathrm{mM} \mathrm{Mg}\left(\mathrm{CH}_{3} \mathrm{COO}\right)_{2}$, and $0.25 \%$ human albumin). The $\mathrm{pH}$ of the fusion medium was adjusted to 7.0 with L-histidine (all chemicals from Sigma-Aldrich). After centrifugation, the cells were resuspended in the same fusion medium in the absence of human albumin. Routinely, $1 \mathrm{ml}$ of cell suspension containing $1 \times 10^{7}$ cells was processed with a specially designed concentric fusion chamber. Electrofusion was carried out using ECM 2001 (BTX Instruments Genetronics, San Diego, $\mathrm{CA}$ ) with a $1-\mathrm{MHz}$ alternative current of $150 \mathrm{~V} / \mathrm{cm}$ for $20 \mathrm{sec}$ following by a direct current pulse of $1200 \mathrm{~V} / \mathrm{cm}$ for $25 \mu \mathrm{sec}$. Five minutes later, the cells were harvested in RPMI-1640 supplemented with $10 \%$ FBS. The next day, adherent cells were harvested as FCs and used for CTL induction.

To determine fusion efficiency, tumor cells were pre-labeled with an intracellular green fluorescent dye, carboxyfluorescein diacetate succinimidyl ester (CFSE) (Molecular Probes, Eugene, OR): $1 \times 10^{7}$ cells/ml in RPMI-1640 were labeled with $5 \mu \mathrm{M}$ dye for $20 \mathrm{~min}$ at $37^{\circ} \mathrm{C}$. After fusion, FCs were detected by staining with CD86 phycoerythrin (PE)-conjugated monoclonal antibody against cell surface marker expressed only by DCs. FCs were double positive on FACS analysis. To confirm fusion efficacy, Giemsa-stained cytocentrifuge preparations were examined for evidence of multinucleated cells, and individual cells were confirmed to show dual fluorescence on confocal microscopy.

CTL induction. CTL induction was conducted by co-culture of PBLs (responder) and four kinds of stimulators in the presence of IL-2 (Shionogi, Osaka, Japan) and IL-7 (Pepro Tech, Rocky Hill, NJ) for 5 weeks (26). Stimulators other 
than FCs included DCs (DCs co-cultured with irradiated tumor cells overnight), Tumor (irradiated tumor-cells alone), and Mix (mixture of DCs and irradiated tumor cells) (Table I). PBLs and stimulators were plated in 24-well plates (Sumiron)

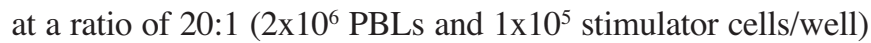
in CTL medium [RPMI-1640 supplemented with $10 \%$ autologous serum, IL-7 (10 ng/ml), and gentamycin $(2 \mu \mathrm{g} / \mathrm{ml})]$. The final volume of the culture medium was $2 \mathrm{ml}$ per well. Cells were harvested and restimulated 3 times at 7-day intervals (days 7,14 , and 21), using the same ratio and numbers of responder and stimulator cells in fresh CTL medium. On days $3,10,17$, and $24,50 \mathrm{U} / \mathrm{ml}$ of IL-2 was added. On days $10,17,24$ and 31 , the medium was half changed with fresh CTL medium. On day 34, induced effector cells were harvested and suspended in fresh CTL medium. Then, $3 \times 10^{6}$ effector cells were plated in each well in preparation for cytotoxic assay. On day 35, the cells were harvested and used for cytotoxic assay and IFN- $\gamma$ release assay.

Cytotoxic assay (europium release assay). Cytotoxic activities of the induced effector cells were measured by europium release assay (27). In vitro cultured target cells [Table I; GT3TKB (human lung cancer cell line), GCIY [human gastric cancer cell line), MKN-45 (human gastric cancer cell line), K-562 (human CML cell line with NK sensitivity)] were harvested and labeled by europium as follows: the cells were washed with buffer A (50 mM HEPES, $93 \mathrm{mM} \mathrm{NaCl}, 5 \mathrm{mM}$ $\mathrm{KCl}$, and $2 \mathrm{mM} \mathrm{MgCl}_{2}, \mathrm{pH} 7.4$; all chemicals from Wako, Osaka, Japan). The cells were then suspended in europium labeling buffer (1 mg europium/1 ml $100 \mathrm{mM}$ DTPH) (Wako) for $20 \mathrm{~min}$ on ice, and the same volume of buffer B (buffer A $+2 \mathrm{mM} \mathrm{CaCl}_{2}, 10 \mathrm{mM}$ glucose; $\mathrm{pH}$ 7.4) (Wako) was added to the medium. Five minutes later, target cells were washed 3 times with buffer B and 2 times with RPMI-1640 containing $10 \%$ FBS. Then, the labeled target cells were resuspended in $100 \mu \mathrm{l}$ of RPMI-1640 containing $10 \%$ FBS $\left(5 \times 10^{4} / \mathrm{ml}\right)$ and distributed into each well of 96 -well $\mathrm{U}$ bottom plates (NUNC, Roskilde, Denmark). Effector cells (20, 10, 5, and $\left.2.5 \times 10^{5} / \mathrm{ml}\right)$ suspended in $100 \mu 1$ of RPMI-1640 containing $10 \%$ FBS were added to the wells (E/T ratios were 40/1, $20 / 1,10 / 1,5 / 1)$. The final volume per well was $200 \mu 1$. The plates were incubated at $37^{\circ} \mathrm{C}$ for $4 \mathrm{~h}$, and the europium concentrations of the supernatants were analyzed with a multilabel counter (Wallac 1420 ARVO, Turku, Finland). Each sample was tested in triplicate. Control wells for determining spontaneous europium release contained only labeled target cells. Maximal release was determined by adding $1 \%$ Triton X-100 (Wako) to the target cells. The percentage of specific lysis was calculated as follows: [(europium release in experimental sample) - (spontaneous europium release]/ [mean maximal release of europium) - (mean spontaneous release of europium)] x 100.

$I F N-\gamma$ release assay. Effector cells were co-cultured with target cells at a ratio of 1:1 in each well of 24-well plates (1x10\% $/$ well effector cells and 1x10\%/well target cells) in RPMI1640 containing $10 \%$ FBS overnight. The final volume of the medium was $2 \mathrm{ml} /$ well. The next day, IFN- $\gamma$ concentrations in the supernatants were measured by enzyme-linked immunosorbent assay (ELISA). Each sample was tested in triplicate.
Table I. Stimulators, responder, and targets used for CTL induction.

Stimulators

1. FCs (fusion cells of DCs and tumor cells)

2. DCs (dendritic cells co-cultured with irradiated tumor cells overnight)

3. Tumor (irradiated tumor cells)

4. Mix. (mixture of DCs and irradiated tumor cells)

5. Cont. (without stimulator cells)

Responder PBLs (non-adherent cells in PBMCs)

Targets

GT3TKB (human lung cancer-cell line) GCIY (human gastric cancer-cell line) MKN-45 (human gastric cancer-cell line) K-562 (human CML cell-line with NK sensitivity)

PBL, peripheral blood lymphocyte; PBMC, peripheral blood mononuclear cell.

CTL induction by FCs with different fusion rates. FCs of DCs and GT3TKB were generated by electrofusion method at three different levels of direct current (data not shown), and FCs with three different fusion rates were obtained. Three groups of CTLs were induced by these FCs with different fusion rates. The cytotoxic activities of these CTLs were evaluated by europium release assay.

Addition of IL-12 to the induction and effector phases of CTLs. To evaluate the effect of IL-12 on in vitro CTL induction, $10 \mathrm{ng} / \mathrm{ml}$ of IL-12 (Pepro Tech, Rocky Hill, NJ) was added to the CTL medium. The cytotoxicity of CTLs induced with IL-12 was compared with that of CTLs induced without IL-12 by europium release assay. In addition, to evaluate whether IL-12 enhances the cytotoxicity of CTLs at the effector phase, two different conditions were used: $10 \mathrm{ng} / \mathrm{ml}$ of IL-12 was either added to the medium used for europium release assay or not added. The cytotoxic activities of CTLs were compared between these two different conditions.

\section{Results}

Phenotypic and morphologic analyses of FCs. Phenotypes of DCs and FCs were analyzed by FACS. DCs used for hybrid cell generation displayed a phenotype characteristic of mature DCs (Fig. 1); they expressed HLA-DR (class II), costimulatory molecules CD80 and CD86, and maturation markers CD40 and CD83. They did not express the monocyte/macrophage marker CD14. GT3TKB and GCIY had none of these molecules on their surface (data not shown). FCs expressed both CFSE of tumor marker and CD86 PE of DC marker (Fig. 2). The fusion rate of adherent cells was $48.5 \%$ for 

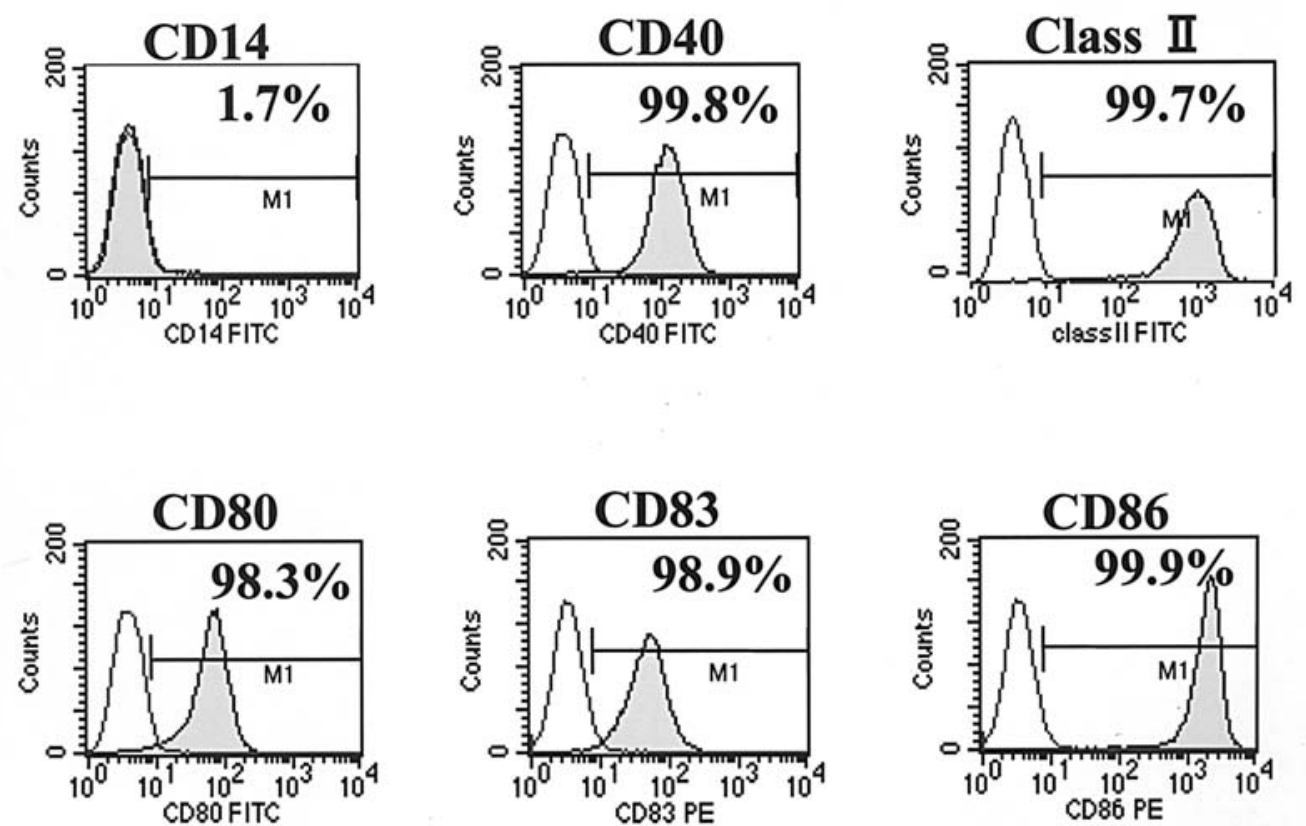

Figure 1. Surface antigens of DCs used for hybrid cell generation. Results of FACS analyses for elutriated DCs on day 7 are shown. Phenotype markers of DCs were analyzed with the following mAbs: anti-CD14, anti-CD40 (Pharmingen), anti-CD80, anti-CD83, anti-CD86, and anti-HLA-DR (class II) (Immunotech, Marseilles, France). Cells were stained either with fluorescent-conjugated monoclonal antibodies directed against the indicated markers or fluorescent-conjugated isotype control antibody. The percent positivity for each marker is indicated.

(a) GT3TKB

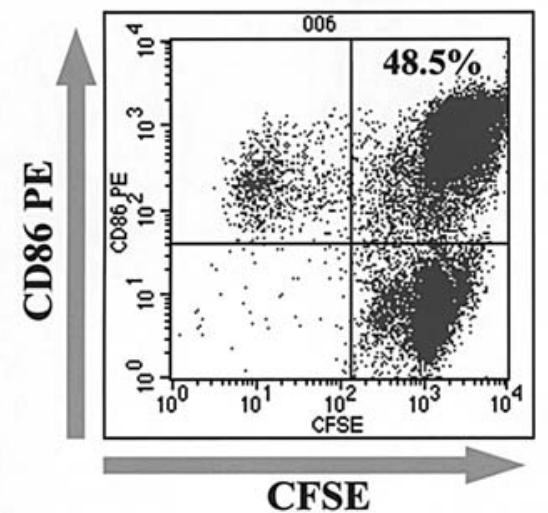

(b) GCIY

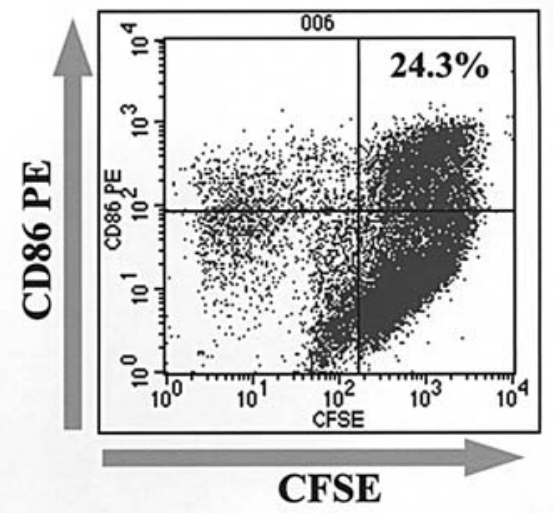

Figure 2. FCs represent both DC markers and tumor cell markers. Results of two-color FACS analyses on FCs of DCs and GT3TKB (lung cancer cell line) (a) or GCIY (gastric cancer cell line) (b) are shown. Both tumor cells were stained with CFSE before fusion. Overnight-cultured adherent FCs were stained with the indicated CD86PE-conjugated mAbs. The percentages of dual-positive cells are indicated in the upper-right quadrant.

GT3TKB and $24.3 \%$ for GCIY. After electrofusion, adherent cells and non-adherent cells were harvested and analyzed by FACS. Adherent cells included more double-positive cells than non-adherent cells (data not shown). Adherent cells were therefore used as FCs.

The morphology of FCs was confirmed by cytocentrifuge preparation and confocal microscopy. Giemsa-stained cytocentrifuge preparations showed large, multinucleated FCs (Fig. 3a and d). On confocal microscopy, the red circumferences (CD86PE) of the cells were derived from DCs, and green cytoplasm (CFSE) was derived from tumor cells. Fig. 3b and e show mixtures of DCs and tumor cells. Fig. $3 \mathrm{c}$ and $\mathrm{f}$ show FCs. FCs had both red circumferences and green cytoplasm.

For GT3TKB, similar fusion rates were obtained on FACS and confocal microscopy, i.e., the rate of double-positive cells was $47.8 \%$ on FACS, and the rate of dual-fluorescent cells was $45.5 \%$ on confocal microscopy (data not shown).

Phenotypes of effector cells induced by FCs. To confirm the phenotypes of PBL-derived effector cells induced by FCs, the cells were dually stained with two mAbs: anti-CD8 mAb 


\section{Giemsa stain}
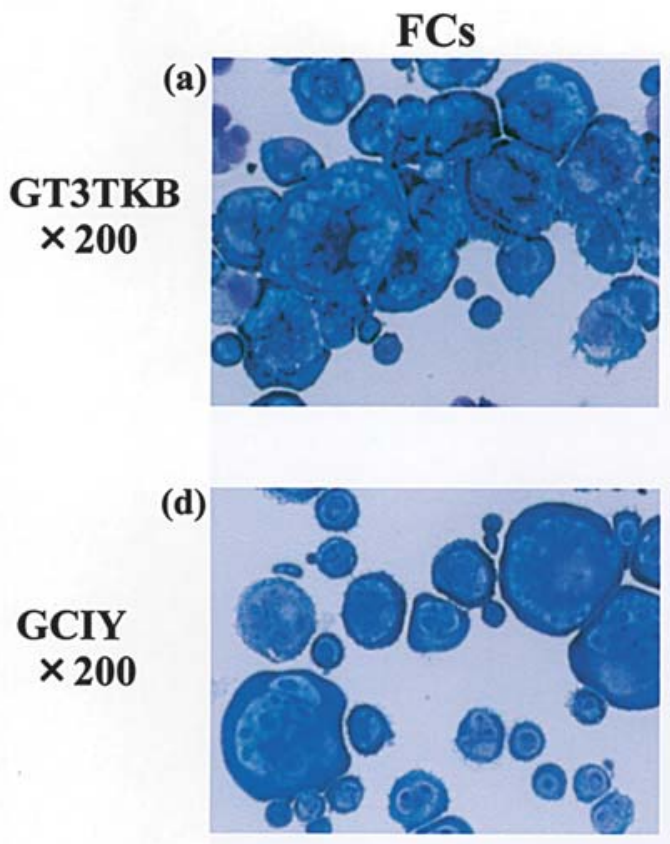

\section{Confocal laser fluorescent microscopy}
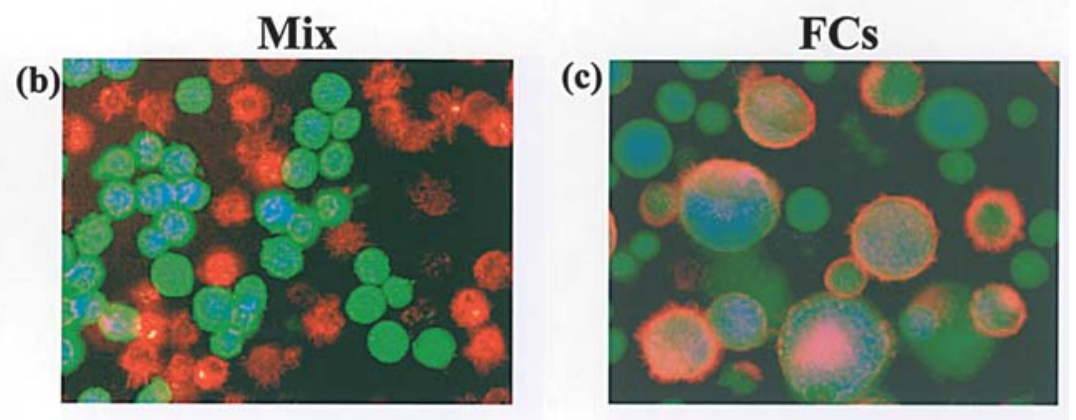

(e)
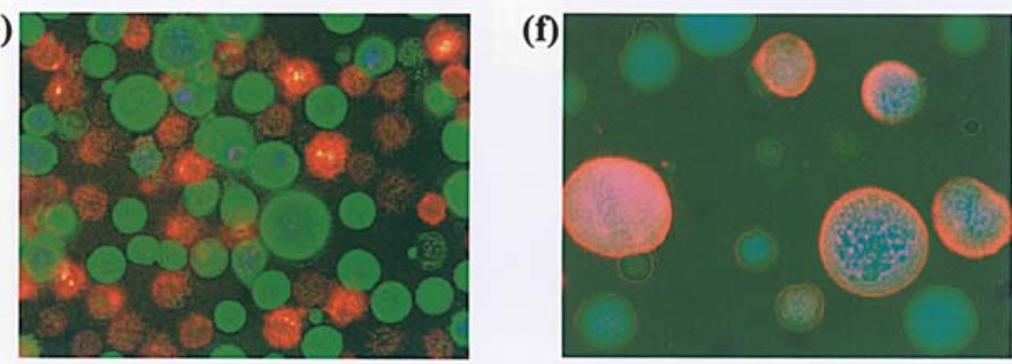

(red : CD 86PE, green : CFSE)

Figure 3. Micrography of FCs. The upper panels (a-c) show FCs when GT3TKB (lung cancer cell line) was used, and the lower panels (d-f) show FCs when GCIY (gastric cancer cell line) was used. (a) and (d) are photographs of Giemsa-stained cytocentrifuge preparations of multinucleated FCs (original magnification, x200). (b), (c), (e) and (f) were obtained by confocal fluorescent microscopy. Green indicates tumor cells labeled with CFSE before fusion, and red indicates DCs labeled with CD86 PE after harvest. (b) and (e) are mixtures of DCs and tumor cells; (c) and (f) are FCs (original magnification, x200).

and anti-CD4 mAb. The results of FACS analyses are shown in Figs. 4a and 5a. The percentage of anti-CD8 mAb positive cells was $96.3 \%$ for GT3TKB (Fig. 4a) and $70.7 \%$ for GCIY (Fig. 5a). Thus, the effector cells induced by FCs were CD8-dominant killer T cells for both cell lines.

In vitro tumor-specific $T$ cell stimulatory activity of FCs. The tumor-specific cytotoxic activities of the effector cells induced by FCs were evaluated by cytotoxic assay. High, tumor-specific cytotoxic activities were observed, and irrelevant targets were killed weakly when GT3TKB was used for generation of FCs (Fig. 4b), as well as when GCIY was used (Fig. 5b). (Fig. 4b-d, FCs were generated from DCs and GT3TKB; Fig. 5b-d, FCs were generated from DCs and GCIY). FCs showed stronger CTL induction capacity than did any other stimulators on both cytotoxic assay and IFN- $\gamma$ release assay (Figs. 4c and d and 5c and d).

Influence of FC fusion rates on CTL induction. FCs of DCs and GT3TKB were generated by our electrofusion method at three different levels of direct current. FCs with three different fusion rates were obtained: fusion rates on FACS were $35.4,42.5$, and $58.7 \%$ respectively. The cytotoxic activities of CTLs induced using FCs with these three different fusion rates did not differ significantly (Fig. 6).

Influence of IL-12 on CTLs in their induction and effector phases. CTLs were induced by co-culture of PBLs and FCs for 5 weeks in the presence of IL-12 [IL-12 (+)] or in the absence of IL-12 [IL-12 (-)]. The cytotoxic activities of these effectors were compared. Addition of IL-12 to the CTL medium had no significant effect on the cytotoxic activities of CTLs (Fig. 7a). The addition of IL-12 also had no effect on the effector phase of CTLs (Fig. 7b).

\section{Discussion}

In this study, FCs were generated by electrofusion from monocyte-derived DCs and human cancer cell lines, with a high fusion rate. We found that FCs are strong stimulators that can induce tumor-specific CTLs from PBLs in vitro. In fact, we were able to induce effector cells with a certain level of antitumor activity by using irradiated tumor cells alone or a mixture of DCs and irradiated tumor cells as stimulators. However, CTLs with higher tumor specificity and antitumor activity were induced when FCs were used as stimulators.

FCs have been generated in two ways: fusion with the use of PEG or electrofusion, the technique used in this study. Many clinical studies of cancer vaccines have used PEGfused cells (28-30). In these studies, the FCs were confirmed to possess the characteristics of both DCs and tumor cells only by FACS analysis. Close inspection of the photographs of the FCs used in these studies frequently suggest cell aggregation rather than fusion. On FACS analysis, however, aggregated cells can be misinterpreted to be FCs expressing specific surface antigens against both DCs and tumor cells. 
(a)

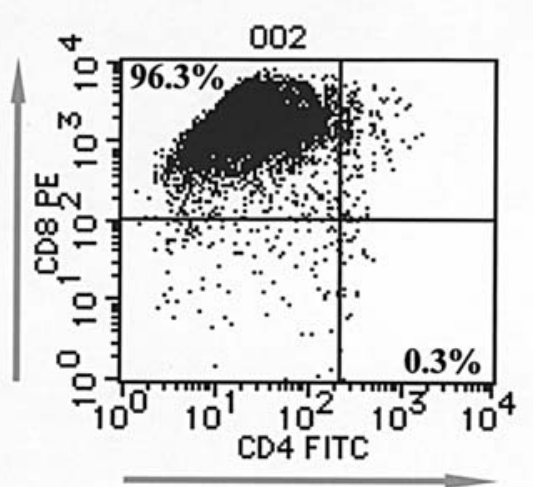

(c)

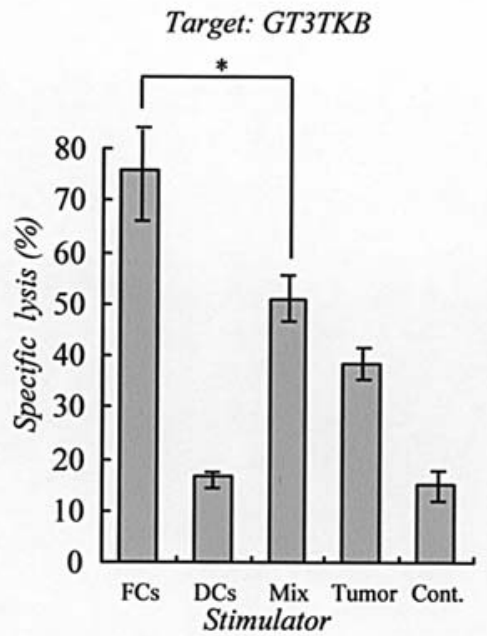

(b)

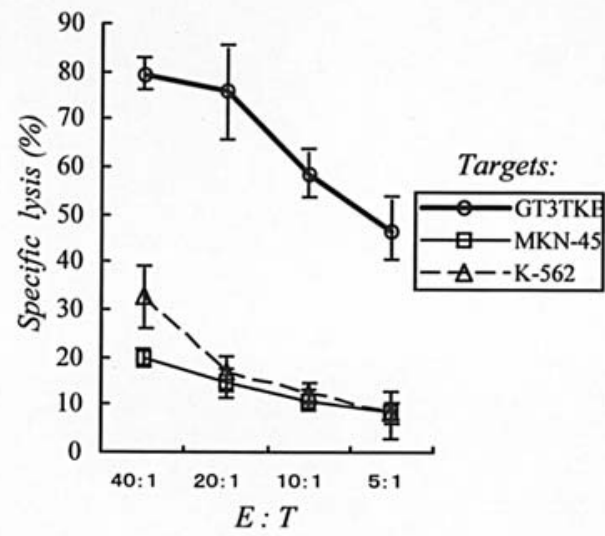

(d)

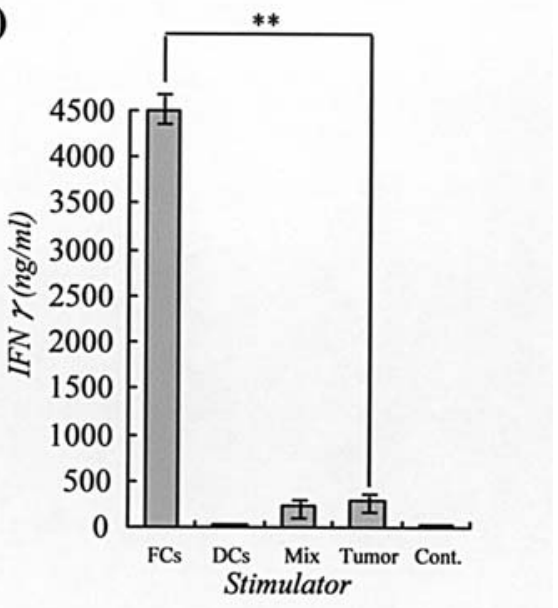

Figure 4. Phenotypes and immune reactivities of effector cells induced by FCs of DCs and GT3TKB (lung cancer cell line). (a) Two-color FACS analysis of effector cells induced by FCs. Percentages of anti-CD8 mAb-positive cells (upper-left quadrant) and anti-CD4 mAb-positive cells (lower-right quadrant) are indicated. (b) Cytotoxic activities of the effector cells induced by FCs. Cytotoxic activities of the effector cells induced by FCs of DCs and GT3TKB against three different targets [GT3TKB $(\mathrm{O})$, MKN-45 $(\square)$, K-562 $(\triangle)$ ] (Table I) were examined by europium release assay. The vertical axis indicates the percent specific lysis, and the horizontal axis indicates the effector to target (E/T) cell ratios. (c) Comparisons of cytotoxic activities of various kinds of effectors induced by different stimulators [FCs, DCs, Mix., Tumor (GT3TKB), Cont.] (Table I). E/T ratio: 20/1. (d) IFN- $\gamma$ release assay. Various kinds of effectors induced by different stimulators [FCs, DCs, Mix., Tumor (GT3TKB), Cont.] (Table I) were co-cultured with irradiated GT3TKB overnight. Thereafter, the IFN- $\gamma$ concentration in each supernatant of culture medium was measured by ELISA. For all samples, mean values and standard deviations of triplicate samples are shown. (Unpaired t-test; ${ }^{*} \mathrm{p}<0.05,{ }^{* * *} \mathrm{p}<0.01$ ).

To our knowledge, no study using PEG fusion has morphologically confirmed cell fusion on confocal fluorescent microscopy and verified that the results were consistent with the findings on FACS, similar to our study. We firmly believe that not only FACS but also morphological confirmation by confocal fluorescent microscopy is necessary to verify whether FCs have been actually generated and to evaluate the fusion efficiency of FCs.

Several studies have previously examined the generation of DC-tumor hybrids by electrofusion technique. Fusion rates have ranged from 14.0 to $62.7 \%$. Our newly developed electrofusion method used in this study can reproducibly generate stable FCs with a high fusion efficiency. Improved fusion efficiency and more consistent results were enabled by adjusting the settings of the fusion devices and modifying the culture medium used for fusion (17). Fusion rates ranged from 31.9 to $58.7 \%$ (mean $44.7 \%$; $\mathrm{n}=11$ trials) for GT3TKB and DCs and from 14.3 to $31.7 \%$ (mean $24.2 \%$; $n=5$ ) for GCIY and DC. In our study, CTLs were induced by using FCs as stimulator cells in vitro to assess the possibility of clinically using FCs for adoptive immunotherapy. Trevor et al also generated FCs from monocyte-derived DCs and Colo-829, a human melanoma-cell line, by electrofusion technique and used the FCs to induce CTLs in vitro (31). With their electrofusion technique, however, the fusion rate was only $10 \%$, clearly lower than that obtained with our electrofusion technique. A high fusion rate may be a very important factor in the induction of CTLs by FCs. We therefore examined whether CTL induction capacity depended on the fusion rate. Our results showed that CTL induction capacity was not significantly altered by different fusion rates (Fig. 6). This finding showed that a high fusion efficiency was not necessarily required for the induction of CTLs by FCs in vitro. This may be explained by the fact that huge FCs with 20-30 nuclei generated 
(a)

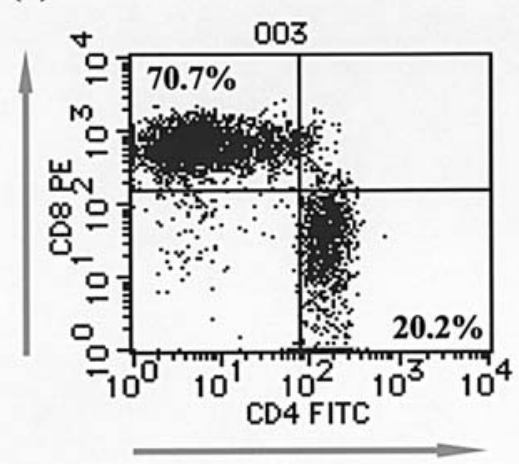

(c)

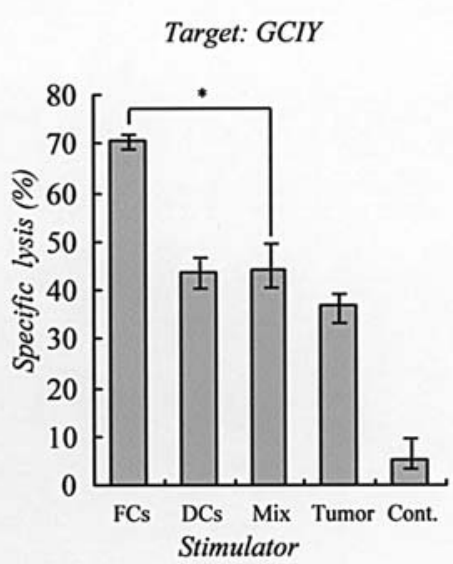

(b)

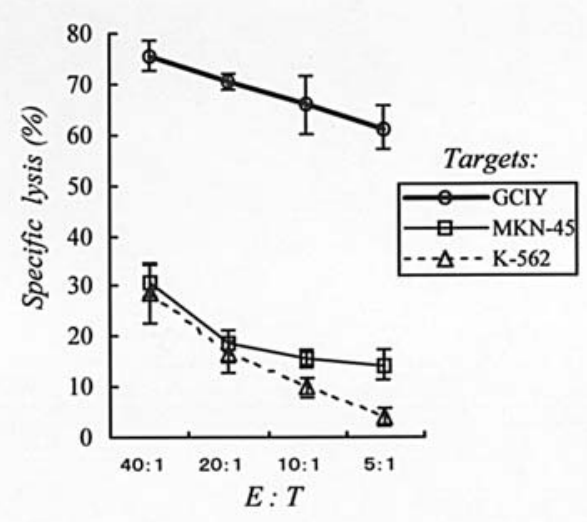

(d)

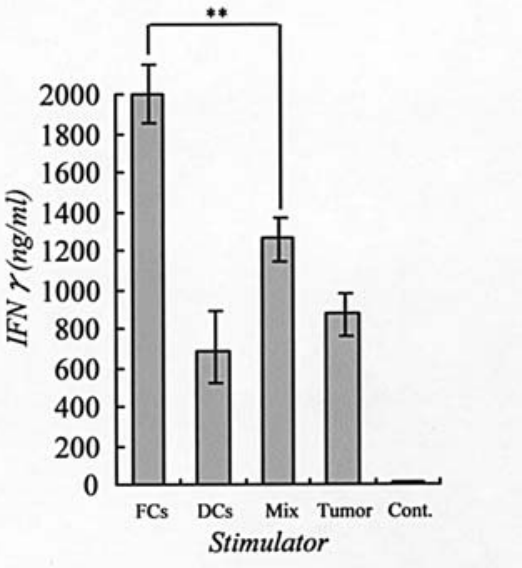

Figure 5. Phenotypes and immune reactivities of effector cells induced by FCs of DCs and GCIY (gastric cancer cell line). (a) Two-color FACS analysis of effector cells induced by FCs. Percentages of anti-CD8 mAb-positive cells (upper-left quadrant) and anti-CD4 mAb-positive cells (lower-right quadrant) are indicated. (b) Cytotoxic activities of the effector cells induced by FCs. Cytotoxic activities of effector cells induced by FCs of DCs and GCIY against three different targets [GCIY $(\mathrm{O})$, MKN-45 $(\square)$, K-562 $(\triangle)$ ] (Table I) were examined by europium release assay. The vertical axis indicates the percent specific lysis, and the horizontal axis indicates the effector to target (E/T) cell ratios. (c) Comparisons of cytotoxic activities of various kinds of effectors induced by different stimulators [FCs, DCs, Mix., Tumor (GCIY), Cont.] (Table I). E/T ratio: 20/1. (d) IFN- $\gamma$ release assay. Various kinds of effectors induced by different stimulators [FCs, DCs, Mix., Tumor (GCIY), Cont.] (Table I) were co-cultured with irradiated GCIY overnight. Thereafter, the IFN- $\gamma$ concentration in each supernatant of culture medium was measured by ELISA. For all samples, mean values and standard deviations of triplicate samples are shown. (unpaired t-test; $\left.{ }^{*} \mathrm{p}<0.05 ;{ }^{* *} \mathrm{p}<0.01\right)$.

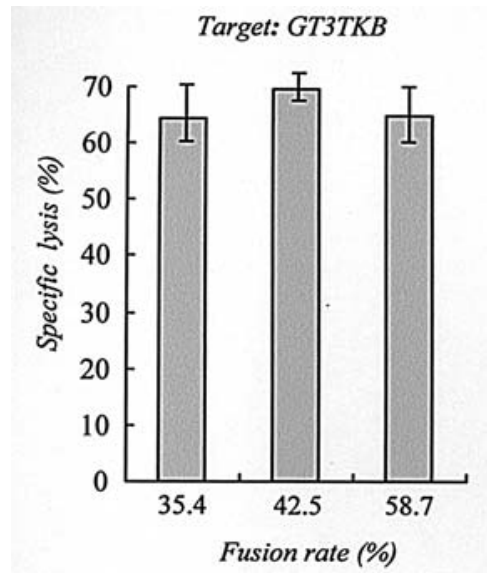

Figure 6. Influence of fusion rates on CTL induction. FCs of DCs and GT3TKB (lung cancer cell line) were generated by electrofusion at three different levels of direct current (data not shown), and three different fusion rates of FCs were obtained (fusion rates as evaluated by FACS: 35.4, 42.5, and $58.7 \%$ respectively). The cytotoxic activities of CTLs induced by these different fusion rates of FCs were evaluated by europium release assay. E/T ratio: 20/1. under conditions favoring high fusion efficiency (data not shown) might not effectively function as APCs.

In experimental studies using tumor-bearing mice given vaccine therapy prepared from FCs, we previously showed that systemic treatment with IL-12 as an adjuvant is necessary for antitumor activity (17-21). IL-12 is a representative antitumor cytokine that activates $\mathrm{T}$ cells and natural killer cells. We and others have reported that IL-12 is a very effective adjuvant for DC-based cancer vaccine therapy in mice (32). In the present study, we examined whether IL-12 promotes the induction of CTLs by FCs in vitro. As shown in Fig. 7, however, IL-12 did not promote the induction of CTLs and did not influence the effector phase, in which CTLs demonstrate cytotoxicity against target cells.

Clinical studies of FCs generated by electrofusion technique have failed to demonstrate adequate antitumor effectiveness $(22,23)$. This lack of efficacy is ascribed to no concomitant use of effective adjuvant agents, such as IL-12, and the presence of factors inhibiting antigen presentation by FCs, i.e., immunosuppressive cytokines in patients with cancer. 

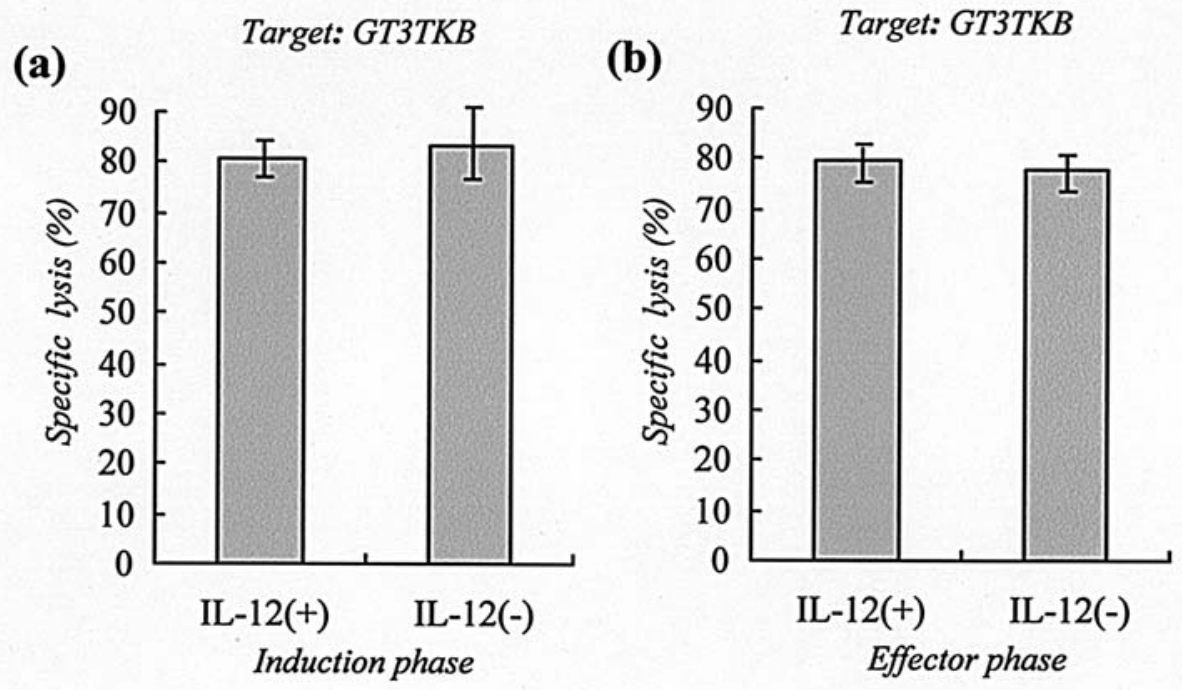

Figure 7. Influence of IL-12 on CTLs in their induction and effector phases. (a) CTLs were induced by co-culture of PBLs and FCs, which were generated by electrofusion of DCs and GT3TKB, for 5 weeks in the presence of IL-12 [IL-12 (+)] or in the absence of IL-12 [IL-12 (-)]. Each CTL was tested by europium release cytotoxic assay (target: GT3TKB, E/T ratio: 20/1). (b) In addition, to evaluate whether IL-12 augments the cytotoxicity of CTLs at the effector phase, CTLs induced without IL-12 were co-cultured with europium-labeled target GT3TKB cells in the presence of IL-12 [IL-12 (+)] or in the absence of IL-12 [IL-12 (-)]. Cytotoxic activities were evaluated by europium release cytotoxic assay (target: GT3TKB, E/T ratio: 20/1).

Kao et al reported that TGF- $\beta$ derived from tumors reduced the efficacy of FC-based vaccine (24). Available evidence suggests that antitumor cytokines such as IL-12 or substances that inhibit immunosuppressive cytokines such as TGF- $\beta$ are required as an adjuvant agent when FCs are used clinically as vaccine for active immunotherapy. However, such adjuvant agents are not necessary when FCs are used to induce CTLs in vitro for clinical use as adoptive immunotherapy.

In conclusion, our study showed that FCs can be consistently generated from monocyte-derived mature DCs and human cancer cell lines by our newly developed electrofusion technique. FCs were shown to act as stimulators in vitro and to efficiently induce tumor-specific CTLs from PBLs. However, it is not easy to establish tumor cell lines from tumor tissue obtained from patients with cancer, except for certain tumor types such as melanoma. Resolution of this problem may lead to the development of systems for the clinical application of FCs generated from patients' own tumor cells.

\section{Acknowledgements}

This work was supported in part by a 2004-2005 Grant-In-Aid from the Ministry of Health, Labor and Welfare, Japan (no. 16241801) (to Y.U.) and a 2003 Grant for R\&D for practical application utilizing the matching fund method from the New Energy and Industrial Technology Development Organization, Japan (to H.Y. and Y.U.). We would like to thank Professor Suyu Shu, Center for Surgery Research, The Cleveland Clinic Foundation, for providing helpful advice on this study.

\section{References}

1. Brossart P, Wirths S, Brugger W and Kanz L: Dendritic cells in cancer vaccines. Exp Hematol 29: 1247-1255, 2001.
2. Steinman R and Nussenzweig M: Dendritic cells; features and functions. Immunol Rev 53: 127-147, 1980.

3. Banchereau J, Brie F, Caux C, Davoust J, Lebecque S, Liu YJ, Pulendran B and Palucka K: Immunobiology of dendritic cells. Annu Rev Immunol 18: 767-811, 2000.

4. Schuler-Thurner B, Schultz SE, Berger GT, Weinlich G, Ebner S, Woerl P, Bender A, Feuerstein B, Fritsch OP, Romani N and Schuler G: Rapid induction of tumor-specific type $1 \mathrm{~T}$ helper cells in metastatic melanoma patients by vaccination with mature, cryopreserved, peptide-loaded monocyte-derived dendritic cells. J Exp Med 195: 1279-1288, 2002

5. Brossart P, Wirths S, Stuhler G, Reichardt LV, Kanz L and Brugger W: Induction of cytotoxic T-lymphocyte responses in vivo after vaccinations with peptide-pulsed dendritic cells. Blood 96: 3102-3108, 2000.

6. Matsuyoshi H, Senju S, Hirata S, Yoshitake Y, Uemura Y and Nishimura Y: Enhanced priming of antigen-specific CTLs in vivo by embryonic stem cell-derived dendritic cells expressing chemokine along with antigenic protein; application to antitumor vaccination. J Immunol 172: 776-786, 2004.

7. Schmitz M, Diestelkoetter P, Weigle B, Schmachtenberg F, Stevanovic S, Ockert D, Rammensee HG and Rieber PE: Generation of survivin-specific $\mathrm{CD} 8^{+} \mathrm{T}$ effector cells by dendritic cells pulsed with protein or selected peptides. Cancer Res 60: 4845-4849, 2000

8. Su Z, Dannull J, Heiser A, Yancey D, Pruitt S, Madden J, Coleman D, Niedzwiecki D, Gilboa E and Vieweg J: Immunological and clinical responses in metastatic renal cancer patients vaccinated with tumor RNA-transfected dendritic cells. Cancer Res 63: 2127-2133, 2003

9. Duane AM and Smita KN: RNA-transfected dendritic cells in cancer immunotherapy. J Clin Invest 106: 1065-1069, 2000.

10. Heiser A, Maurice AM, Yancey RD, Coleman MD, Dahm P and Vieweg J: Human dendritic cells transfected with renal tumor RNA stimulate polyclonal T-cell responses against antigens expressed by primary and metastatic tumors. Cancer Res 61: 3388-3393, 2001

11. Yu SJ, Liu G, Ying H, Yong HW, Black LK and Wheeler JC: Vaccination with tumor lysate-pulsed dendritic cells elicits antigen-specific, cytotoxic T-cells in patients with malignant glioma. Cancer Res 64: 4973-4979, 2004.

12. Hus I, Rolinski J, Tabarkiewicz J, Wojas K, Bojarska-Junak A, Greiner J, Giannopoulos K, Dmoszynska A and Schmitt M: Allogeneic dendritic cells pulsed with tumor lysates or apoptotic bodies as immunotherapy for patients with earlystage B-cell chronic lymphocytic leukemia. Leukemia 19: 1621-1627, 2005. 
13. Stift A, Friedl J, Dubsky P, Bachleitner-Hofmann T, Schueller G, Zontsich T, Benkoe T, Radelbauer K, Brostjan C, Jakesz R and Gnant M: Dendritic cell-based vaccination in solid cancer. J Clin Oncol 21: 135-142, 2003.

14. Candido AK, Shimizu K, McLaughlin CJ, Kunkel R, Fuller AJ, Redman GB, Thomas KE, Nickoloff JB and Mule JJ: Local administration of dendritic cells inhibits established breast tumor growth; implications for apoptosis-inducing agents. Cancer Res 61: 228-236, 2001

15. Zhou Y, Bosch ML and Salgaller ML: Current methods for loading dendritic cells with tumor antigen for the induction of antitumor immunity. J Immunother 25: 289-303, 2002.

16. Laskov R, Kim JK and Asofsky R: Induction of amplified synthesis and secretion of IgM by fusion of murine B lymphoma with myeloma cells. Proc Natl Acad Sci USA 76: 915-919, 1979.

17. Hayashi T, Tanaka H, Tanaka J, Wang R, Averbook JB, Cohen AP and Shu S: Immunogenicity and therapeutic efficacy of dendritic-tumor hybrid cells generated by electrofusion. Clin Immunol 104: 14-20, 2002.

18. Tanaka H, Shimizu K, Hayashi T and Shu S: Therapeutic immune response induced by electrofusion of dendritic and tumor cells. Cell Immunol 220: 1-12, 2002.

19. Kuriyama H, Shimizu K, Lee W, Kjaergaard J, Parkhurst MR, Cohen PA and Shu S: Therapeutic vaccine generated by electrofusion of dendritic cells and tumour cells. Dev Biol 116: 169-178, 2004.

20. Shimizu K, Kuriyama H, Kjaergaard J, Lee W, Tanaka H and Shu S: Comparative analysis of antigen loading strategies of dendritic cells for tumor immunotherapy. J Immunother 27: 265-272, 2004.

21. Lee TW, Shimizu K, Kuriyama H, Tanaka H, Kjaergaard J and Shu S: Tumor-dendritic cell fusion as a basis for cancer immunotherapy. Otolaryngol Head Neck Surg 132: 755-764, 2005.

22. Haenssle AH, Krause WS, Emmert S, Zutt M, Kretschmer L, Schmidberger H, Andreesen R and Soruri A: Hybrid cell vaccination in metastatic melanoma clinical and immunologic results of a phase I/II study. J Immunother 27: 147-155, 2004

23. Trefzer U, Herberth G, Wohlan K, Milling A, Thiemann M, Sharav T, Sparbier K, Sterry W and Walden P: Tumor-dendritic hybrid cell vaccination for the treatment of patients with malignant melanoma: immunological effects and clinical results. Vaccine 23: 2367-2373, 2005.
24. Kao YJ, Gong Y, Chen MC, Zheng DQ and Chen JJ: Tumorderived TGFß reduces the efficacy of dendritic cell/tumor fusion vaccine. J Immunol 170: 3806-3811, 2003.

25. Lee AW, Truong T, Bickham K, Fonteneau JF, Larsson M, Da SI, Somersan S, Thomas EK and Bhardwaj N: A clinical grade cocktail of cytokines and PGE2 results in uniform maturation of human monocyte-derived dendritic cells: implications for immunotherapy. Vaccine 20: A8-A22, 2002.

26. Baxevanis NC, Voutsas FI, Tsitsilonis EO, Gritzapis DA, Sotiriadou R and Papamichail M: Tumor-specific CD4 ${ }^{+} \mathrm{T}$ lymphocytes from cancer patients are required for optimal induction of cytotoxic $\mathrm{T}$ cells against the autologous tumor. $\mathrm{J}$ Immunol 164: 3902-3912, 2000.

27. Uherek C, Tonn T, Uherek B, Becker S, Schnierle B, Klingemann HG and Wels W: Retargeting of natural killer-cell cytolytic activity to ErbB2-expressing cancer cells results in efficient and selective tumor cell destruction. Blood 100: 1265-1273, 2002.

28. Gong J, Nikrui N, Chen D, Koido S, Wu Z, Tanaka Y, Cannistra S, Avigan D and Kufe DJ: Fusions of human ovarian carcinoma cells with autologous or allogeneic dendritic cells induce antitumor immunity. Immunology 165: 1705-1711, 2003.

29. Kikuchi T, Akasaki Y, Irie M, Homma S, Abe T and Ohno T: Results of a phase I clinical trial of vaccination of glioma patients with fusions of dendritic and glioma cells. Cancer Immunol Immunother 50: 337-340, 2001.

30. Krause SW, Neumann C, Soruri A, Mayer S, Peters JH and Andreesen R: The treatment of patients with disseminated malignant melanoma by vaccination with autologous cell hybrids of tumor cells and dendritic cells. J Immunother 25: 421-428, 2002.

31. Trevor TK, Cover C, Ruiz WY, Akporiaye TE, Hersh ME, Landais D, Taylor RR, King DA and Walters ER: Generation of dendritic cell-tumor cell hybrids by electrofusion for clinical vaccine application. Cancer Immunol Immunother 53: 705-714, 2004.

32. Tatsumi T, Takehara T, Kanto T, Miyagi T, Kuzushita N, Sugimoto Y, Jinushi M, Kasahara A, Sasaki Y, Hori M and Hayashi N: Administration of interleukin-12 enhances the therapeutic efficacy of dendritic cell-based tumor vaccines in mouse hepatocellular carcinoma. Cancer Res 61: 7563-7567, 2001. 